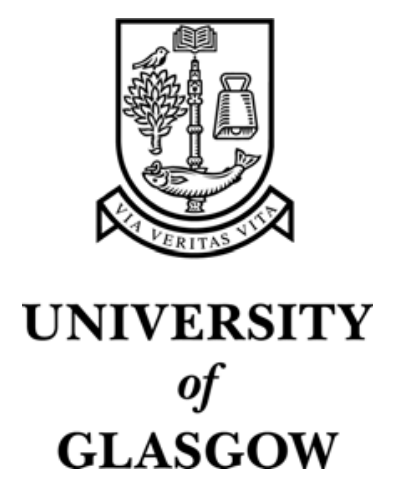

Yu, W. and Ramloll, R. and Brewster, S.A. (2001) Haptic graphs for blind computer users. Lecture Notes in Computer Science 2058:pp. 41-51.

http://eprints.gla.ac.uk/3212/ 


\title{
Haptic Graphs for Blind Computer Users
}

\author{
Wai Yu, Ramesh Ramloll and Stephen Brewster \\ Department of Computing Science \\ University of Glasgow \\ Glasgow, G12 8QQ, UK \\ +44 1413304966 \\ rayu@dcs.gla.ac.uk \\ ramesh@dcs.gla.ac.uk \\ stephen@dcs.gla.ac.uk \\ http://www.dcs.gla.ac.uk/ stephen
}

\begin{abstract}
In this paper we discuss the design of computer-based haptic graphs for blind and visually impaired people with the support of our preliminary experimental results. Since visual impairment makes data visualisation techniques inappropriate for blind people, we are developing a system which can make graphs accessible through haptic and audio media. The disparity between human haptic perception and the sensation simulated by force feedback devices is discussed. Our strategies to tackle technical difficulties posed by the limitations of force feedback devices are explained. Based on the results of experiments conducted on both blind and sighted people, we suggested two techniques: engraving and the use of texture to model curved lines on haptic graphs. Integration of surface property and auditory cues in our system are proposed to assist blind users in exploring haptic graphs.
\end{abstract}

\section{Keywords}

Haptics, haptic graphs, assistive technology, visualization, multimodal interaction

\section{INTRODUCTION}

We are currently conducting an EPSRC funded project (Multivis) aimed at providing access to complex graphical data, i.e. graphs and tables, for blind and visually impaired people. A multimodal approach, using sound and touch, is adopted in this research. Traditionally, graphs and diagrams are presented in Braille, and raised dots and lines on the swell-paper. Several problems are associated with this kind of graph presentation technique. Firstly, only a small proportion of blind people has learned and uses Braille (only $26 \%$ of blind university students use it). Secondly, the resolution and the accuracy of the raised graphs and diagrams is fairly low so that blind people can only get a rough idea about the content. Thirdly, complex details on the graph are difficult to perceive and become rather confusing. Fourthly, dynamic data, which could change frequently, cannot be represented by the traditional approach. Finally, no assistance is available to blind people when exploring the graph so that this process becomes quite time consuming and tiring. Therefore, we would like to tackle these problems by using computer technology, such as force feedback devices, 3D sound and computer assistance to help blind and visually impaired people to access graphs and diagrams. In this paper we focus on the current state of our research and discuss future work on haptic graphs.

\section{Haptic Perception}

Touch or haptic perception is usually considered as a secondary sensory medium to sighted people although it is very important in our daily lives. We need touch feedback to manipulate objects successfully and effectively, for instance grasping a cup of coffee and turning the door handle. Touch is even more crucial for visually impaired people and become their primary sensory feedback. Haptic receptors are located all over our body and have been classified into two main categories: cutaneous and kinesthetic. Cutaneous receptors reside beneath the surface of the skin and respond to temperature, pain and pressure. Kinesthetic receptors are located in muscles, tendons and joints, and correspond to the position of limbs and their movement in space [Klatzky \& Lederman, 1999].

\section{Touch and Force Feedback Devices}

Force feedback devices are available to provide a haptic channel of information exchange between humans and computers. Ramstein et al. have developed the PC-Access system which offers auditory information (non-verbal sounds and voice synthesis) reinforced by the sense of touch via a force feedback device called the Panograph to enhance users' productivity, increase their satisfaction and optimise their workload [Ramstein et al., 1996]. More recently, Grabowski and Barner have investigated the use of a combination of the sense of touch, using the PHANToM haptic device, and representative soundscapes to develop visualisation aids for blind and visually impaired individuals [Grabowski and Barner, 1998] .

Most current force feedback devices can provide movements in either 2D or 3D space. Commonly, users need to insert one of their fingers into a thimble or hold a 
stylus attached to one end of the mechanical linkage, which is coupled with electrical motors at the other end, to feel the reaction force (Figure 1). By tracking the position of the thimble or the tip of the stylus, contact and interaction with virtual objects can be represented by the appropriate force generated from the motors. Therefore, force feedback devices are good at simulating kinesthetic sensory information but not at cutaneous sensation [Oakley et al. 2000]. Only a single point contact can be produced which means that only the interaction force corresponding to users' finger-tip is simulated by the devices. This is a huge bandwidth reduction on the haptic channel when compared with the number of haptic receptors in human body. By using force feedback devices, people can manipulate virtual objects, and feel their shape and weight. However, detailed and realistic texture on an object is difficult to reproduce due to the limitation of single point contact.

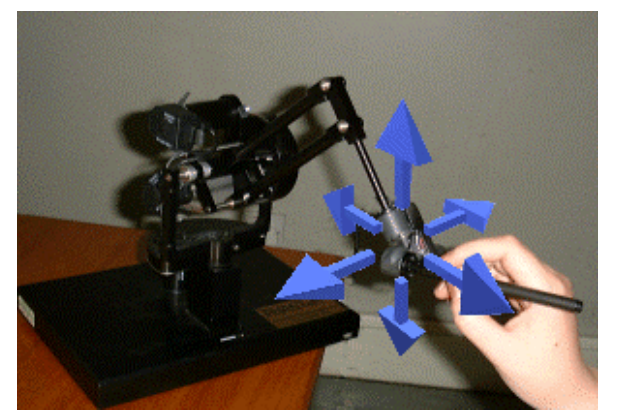

Figure 1. A PHANTOM device with overlaid arrows showing all possible movements. (PHANToM is a product of SensAble Technologies, Inc.)

This limitation in the force feedback devices has a major effect on our haptic graph representation. Braille, and raised dots and lines used in the tactile graphs rely on users' sensitive and rich cutaneous receptors in the fingers. By pressing a finger on the embossed objects, the skin is deformed and gives a tactile perception of the shape and size of the objects. However, in the virtual haptic graphs, users only have a single point contact which will not give instant information about the property of the object being contacted. Therefore, constructing graphs by using embossed objects can cause various problems which will be explained in the following sections.

\section{PRELIMINARY STUDIES}

Several pilot studies have been conducted in the Department of Computing Science at the University of Glasgow [Pengelly, 1998]. These studies were to investigate the use of a force feedback device (PHANToM) to provide haptic representation of line graphs and bar charts. The main areas investigated include: (1) whether users can obtain general information about the graph, (2) effect of haptic gridlines on providing data values on the graph, and (3) whether users can distinguish different lines based on various levels of surface friction. The results have shown that users were able to get a general idea about the layout of the graph through the developed haptic interface. However, not all the users were able to use the gridlines to find the maximum and minimum points on the lines. Moreover, some users found them disturbing when exploring the graphs. The effect of different friction levels on the lines was not obvious because some users had problems distinguishing the difference. Users were often confused when exploring complex graphs and as a result an incorrect layout of the graph was perceived. This is undesirable and contradicts the aim of the haptic interface which is supposed to be an aid to blind people.

Therefore, the haptic interface has been modified and an experiment was conducted to investigate the effect of the change on haptic graph exploration [Flisch, 1999]. The levels of friction were reduced into two: sticky and slippery. A toggling feature was implemented on haptic gridlines so that users can turn them on/off whenever they like. The hypotheses here were:

- The distinctive friction key can be used to distinguish separate lines on the graphs.

- Toggled gridlines will provide an effective means of measurement and reference within the graphs.

\section{Experiment Set Up}

Several line graphs were created for this experiment (Figure 2). In common, two lines were displayed on a graph and they were either located separately or crossintersecting each other. Two different friction properties were applied to the lines and they were classified as sticky and slippery. The lines were made up by cylinders, which are one of the primitive shapes supported by the GHOST programming SDK. Therefore, straight-line approximation was used to construct simple line graphs. All the lines were half sub-merged into a flat surface and thus a semicircle cross-section was formed on all the line models. Due to the circular cross-section of cylinders, users can feel the jagged corner at the joints of the graph lines.
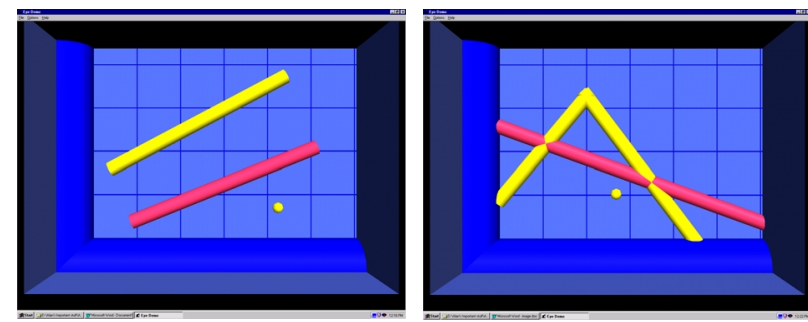

Figure 2 Examples of the graphs used in the experiments. (They show the sticky and slippery lines, $\mathrm{X}$ and $\mathrm{Y}$ axes, and gridlines.)

Both sighted and blind people were recruited as participants for this experiment. Ten sighted participants were used and nine were male. Most of them were from a postgraduate course in I.T. Their ages range from 20 to 35. 
Five blind participants were invited from a local organization for blind people. Their ages were between 30 and 71 and they had different education backgrounds. It was hoped that ten blind participants could have been obtained but only five participants volunteered to take part in the event. Such a small number is insufficient to provide any statistically meaningful results but some implications can still be obtained from the experiment results.

Training was given to the participants to familiarize them with the PHANToM device and the features of the graphs. They had one minute on each of the following graphs, (1) a blank graph, (2) a graph with two parallel lines and (3) a graph with intersecting lines. Therefore they were introduced to the general layout of the graphs, friction key, toggled gridlines and the potential problems of jagged corners. The sighted participants were not allowed to see the graphs on the computer screen throughout the training and experiment sections.

The experiment was divided into two parts. Part 1 was designed to test the friction key. Participants had to explore two graphs in one minute each. Each graph had two parallel lines. At the end, participants needed to identify the sticky and slippery lines and the steeper of the two lines on each of the two graphs.

Part 2 was concerned with testing the toggled gridlines as well as further testing the friction key and general perception of the graphs. Sighted and blind participants had different designs of the experiment procedures and measurements. For the sighted participants, four minutes were given on each of the six graphs which had crossintersected lines. During the four minute exploration, sighted participants needed to obtain the co-ordinates of the maximum and minimum points of each line based on the gridlines. After the exploration, participants were asked to make a sketch of the perceived graph. For the blind participants, only four graphs were given with six minutes exploration time on each. This was because the number of measurements was increased and the experiment had to be kept to a reasonable length. Sketches were not required but participants had to identify and estimate the $\mathrm{X}$ and $\mathrm{Y}$ coordinates of the maximum and minimum values of each line. They were also asked to determine the locations where the lines crossed the axes (if different from the $\mathrm{max} / \mathrm{min}$ co-ordinates) and the crossover points if there was any time left.

All the cursor activities, which are the movements of the PHANToM's stylus, were recorded and saved into a $\log$ file. They provided the traces of participants' behaviour during the graph exploration and thus became useful at the stage of data analysis.

After completing parts 1 and 2, all the participants were asked to fill out a questionnaire which was concerned with four areas:

- The perceived difficulty of the tasks
- The effectiveness of the gridlines

- The usefulness of the toggling gridlines feature

- The effectiveness of the friction key

Participants were asked to rate each area on a scale of one to ten and give any comments on the experiment and the interface in general.

\section{Results}

In the thirty tests carried out on both sighted and blind participants to distinguish the lines by their surface friction, 93.3\% of the responses were correct (Figure 3). Large variation was found on participants' feedback on the questionnaire (Figure 4). The mean rating is 6.5. This difference could be because the usefulness of the friction key was hindered by other factors, such as complexity of the graphs and the line modeling technique. Despite this difference, the friction key was effective at indicating different lines on a simple graph, provided there are no other sources of confusion.



Figure 3. Correct Distinction of Lines according to Friction in Part 1 (sighted participants: 1-10; blind participants 11-15).

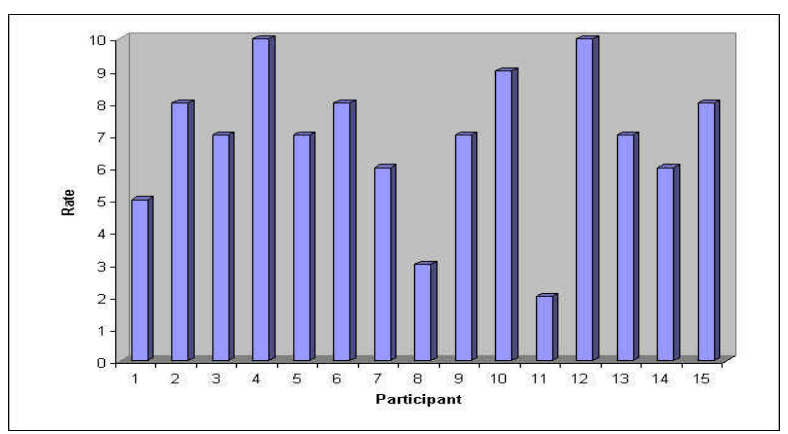

Figure 4. Participants' rating on the effectiveness of the friction key (sighted participants: 1-10; blind participants 11-15).

No conclusive results supported the effectiveness of the gridlines which was judged on the participant's performance in estimating the maximum and minimum values of the graph lines. Sighted participants' rating on the questionnaire matched their performance. However, 
blind participants gave a very high rating even though the majority performed poorly. The mean rating of the effectiveness of the gridlines was 7 out of 10 (Figure 5). The result showed that when participants were confident of the shape and layout of the graphs then the gridlines could be used effectively. However counting the gridlines is often affected by the obstruction of other lines on the graph.



Figure 5. Participants' rating on the effectiveness of the gridlines (sighted participants: 1-10; blind participants 11-15).

Sighted and blind participants had different ratings on the effectiveness of toggling gridlines (Figure 6). Nine out of ten sighted participants rated its effectiveness as 8 or greater out of 10 . On the other hand, three blind participants chose not to use the toggling feature and thus no conclusion can be drawn. However, it was noticeable that the participants who made most use of it tended to produce the most accurate results.

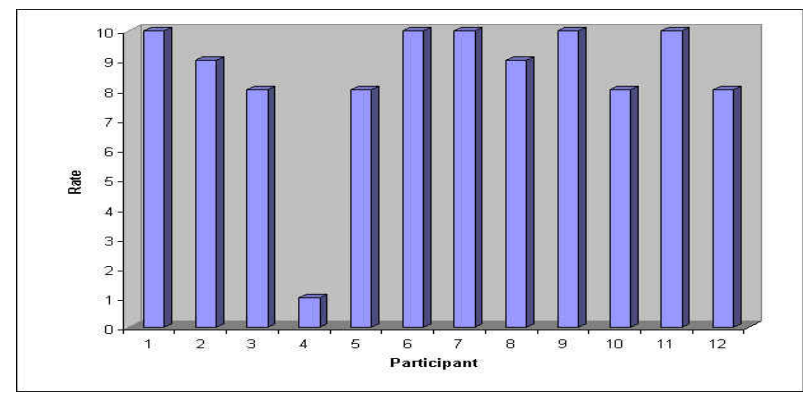

Figure 6. Participants' rating on the usefulness of the toggling gridlines (sighted participants: 1-10; blind participants 11-15).

\section{Discussion}

Three main issues were investigated in the experiment. Using friction key was shown to be useful to distinguish different lines on a simple haptic graph but become less effective in a complex graph. Some participants were confused by the cross intersection points between two lines. They thought the graph consisted of two separate lines combining sticky and slippery parts. This can be seen from the sketch drawn by the participants after the graph exploration (Figure 7). Effect of the friction key was hindered by the embossed cylindrical lines. The line modeling technique, which simply joined cylinder objects together, caused this confusion. Participants found it hard to keep their pointer on the line, especially at the corners and the end points of the lines (Figure 8). This increased the difficulty of tracing the trend of the lines and instead participants struggled to figure out the shape of the line model. Therefore, this simple line modeling technique cannot provide users with effective graph exploration but obstructs users from perceiving correct information about the graph. Different line modeling techniques which can produce curved lines and smooth joints are required.



(a)

(b)

Figure 7. Comparison of (a) participant perceived graph and (b) the actual graph.

Gridlines provide an aid to find the values on a haptic graph. However, the experiment results showed that they were not very effective and not every participant could use them easily. There are four fundamental problems associated with this kind of haptic gridlines. First of all, the uneven surface caused by the raised gridlines may distract users from searching the data lines. Secondly, gridlines are often overlapped by data lines and thus become very difficult to count. Thirdly, they only provide approximate values which are not so useful when exact values are required. Finally, it is very time consuming to count the gridlines because users need to remember the counted numbers in cases of comparing different points on 
the lines. Therefore, it is very difficult to provide exact values of the points on graphs through the haptic interface. Another sensory modality e.g. sound, is needed to solve this problem. Synthesized speech could be used to speak out the value when users press a key on the keyboard or the switch on the PHANToM's stylus.

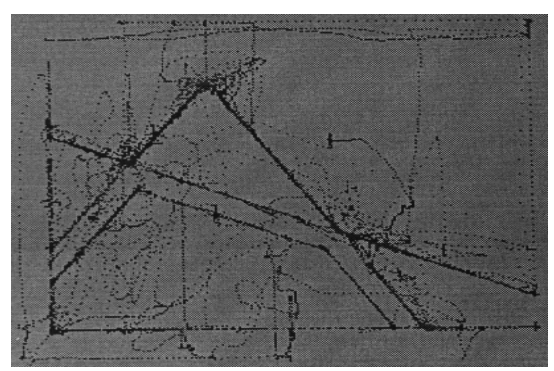

(a)

(b)

Figure 8 (a) Cursor log of a participant's exploration trace. Problems at corners are represented by the chaotic trace of the cursor position. (b) The graph explored by the participant.

Although the number of blind participants involved in the experiment is quite small, it did raise some issues on choosing participants for future experiments. Blind people's age, educational background and knowledge of mathematics and graphs may affect their performance in the experiment. Moreover, perception of graphs may varies from people blind from birth and people blind in the later stage of their life. Therefore, the experimenter needed to explain $\mathrm{x} \& \mathrm{y}$ axes and co-ordinate values to participants who have congenital blindness and have not much knowledge on graphs. On the other hand, an adventitiously blind participant managed to explore the graphs quickly and locate the maximum and minimum values accurately. Therefore, a homogenous group of blind participants, who are of a similar age and have similar experience on graphs, will be required to test or to evaluate further the effectiveness of the haptic interface.

\section{FUTURE WORK}

Issues of future research are raised based on the implications obtained from the preliminary studies:
- Developing different techniques to model curved lines;

- Solving user confusions at intersection points between several lines;

- Using surface friction/texture to distinguish multiple lines;

- Investigating methods to provide a quick overview of graphs;

- Incorporating other sensory modalities into the haptic interface.

As shown in the experiment's results, the line modeling technique using cylinder objects, which are simply jointed together, does not give users a smooth sensation at the joints. The single point contact given by PHANToM also contributes to this problem because the user's pointer cannot stay on the surface of the cylindrical objects easily. It clearly shows that traditional emboss technique used to present text and diagrams to blind people is not suitable for force feedback devices. Instead, an engraving technique is proposed here to present line graphs on the haptic interface. Curved lines can be represented by a groove on a flat surface so that users can easily locate and follow the track of the groove (Figure 9). Techniques of modeling and joining this kind of groove segments by polygons have been developed. Initial testing showed this technique is effective and can solve the problems stated above. However, further improvement is needed in order to handle multiple lines.

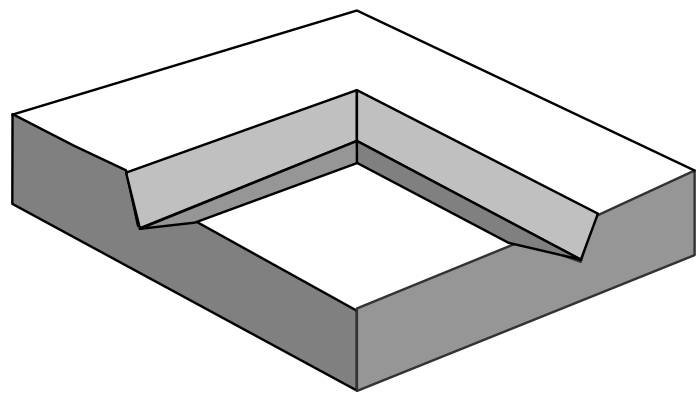

Figure 9. Engraved line on a flat surface.

The problem with intersections between multiple lines is that users get confused when they reach the crossover points. They may lose their sense of direction at the junction where two lines intersect. There are various ways to solve this problem. All the lines on the graph can be displayed selectively, therefore when the user's pointer is moving in a groove, the other lines can be automatically hidden from the user so that smooth transitions can be provided. Alternatively, different textures can be applied on the surfaces of the grooves so that users can tell which groove they are supposed to follow by distinguishing the different sensation. In addition, sound hints can be 
produced by giving an auditory feedback when users switch between grooves.

There are many different parts on a graph so that various surface textures can be applied in order to tell them apart. Since preliminary results have shown that users can distinguish different frictions applied on the lines, mixtures of friction and texture can be used as a distinctive feature of an object. Using surface texture not only can solve the confusion of multiple lines but also gives an indication of different parts of the graph so that users will know where they are on the graph. Investigation is needed here to identify which type of texture can be easily perceived by users via the PHANToM because force feedback devices are generally not good at presenting cutaneous sensation [Oakley et al. 2000].

When blind people are exploring an unknown object, they often want to know the outline of the object by touching it. The same situation applies to graph exploration where blind users would like to know the boundary and dimensions of the graph before feeling the other objects in detail. As the limitation of single point contact, information received by blind users is fairly localised and restricted at that instant of time. Therefore, blind users need to spend a longer time in order to get a general idea about the layout of the line graph. An effective way of presenting the overview of the line graph will shorten the time required in this process and give blind users a better understanding about the graph. Using non-speech sound to provide this kind of quick overview are being investigated.

Using force feedback devices opens a door to graph access for blind people, however, it has some limitations, such as low accuracy and limited bandwidth, so that some information cannot be represented in haptics effectively. For blind people, hearing is another important sensory medium therefore auditory feedback can be introduced into the haptic interface to present information either explicitly by using synthesized speech or implicitly using non-speech sounds. The multimodal approach is used in this research project to provide blind people with access to graphical information.

\section{CONCLUSION}

In this paper, we introduced our research work on developing a multimodal system to make graphs accessible to the blind and visually impaired people. Some preliminary studies have been carried out to evaluate the usefulness of the PHANToM force feedback device in providing this haptic information. Issues in presenting line graphs on the developed haptic interface were discussed with the support of the results obtained from experiments. The PHANToM has been proved to be good at providing kinesthetic rather than cutaneous sensation. The single point contact provided by PHANToM is inadequate when used on embossed line graph objects. Therefore, different line graph modeling techniques, which engrave data lines on a flat surface, have been developed to solve this problem. Friction and surface textures were shown to be useful to distinguish different objects on the line graph, whereas, toggled gridlines were unable to provide approximate values on the lines easily to the users. Users could get a general idea about the layout of the line graph through the developed haptic interface. However, it also indicated that the line graph perceived by the users is often distorted and inaccurate due to the limitations of the force feedback device and the modeling technique. The implications of the preliminary studies have inspired the future development of this research work. The issues of presenting line graphs more effectively to blind people were discussed. In conclusion, haptic interface are useful to provide graph information to blind computer users, however, its limitations indicate that a multimodal approach would be more appropriate and effective for our work.

\section{ACKNOWLEDGMENTS}

The authors would like to thank the Glasgow and Western Scotland Society of Blind for its participation in the experiments. This research work is funded by EPSRC Grant GR/M44866, ONCE (Spain) and Virtual Presence Ltd.

\section{REFERENCES}

Flisch A. (1999). Investigation of the Effectiveness of a Haptic Graph Interface, Masters dissertation, Department of Computing Science, University of Glasgow, 1999.

Grabowski, N. A. and K. E. Barner (1998). Data Visualisation Methods for the Blind Using Force Feedback and Sonification. Part of the SPIE Conference on Telemanipulator and Telepresence Technologies V, Boston Massachusetts, pp 131-139.

Klatzky, R.L. \& Lederman, S.J. (1999). The haptic glance: A route to rapid object identification and manipulation. In D. Gopher \& A. Koriats (Eds.) Attention and Performance XVII. Cognitive regulations of performance: Interaction of theory and application. (pp. 165-196). Mahwah, NJ: Erlbaum.

Oakley, I., McGee, M.R., Brewster, S.A. and Gray, P.D. (2000). Putting the feel in look and feel. In ACM CHI 2000 (The Hague, NL), ACM Press Addison-Wesley, pp 415422.

Pengelly H. L. (1998). Investigating the Use of Forcefeedback Devices in Human-Computer Interaction, Masters dissertation, Department of Computing Science, University of Glasgow, 1998.

Ramstein C, Martial O, Dufresne A, Carignan M, Chasse P, Mabilleau P. (1996). Touching and Hearing GUI's: Design Issues for the PC-Access System. Second Annual ACM Conference on Assistive Technologies, pp 2-9. 